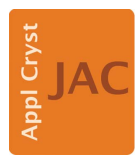

JOURNAL OF APPLIED CRYSTALLOGRAPHY

ISSN 1600-5767

Received 12 March 202

Accepted 20 April 2021

Edited by T. J. Sato, Tohoku University, Japan

Keywords: pole figures; neutrons; characterization.

\section{Alignment facility and software for single-crystal time-of-flight neutron spectroscopy}

\author{
Zihao Liu, ${ }^{a}$ Harry Lane,,${ }^{\text {a,b,c }}$ Christopher D. Frost, ${ }^{\mathrm{c}}$ Russell A. Ewings, ${ }^{\mathrm{c}}$ J. Paul \\ Attfield $^{\mathrm{b}}$ and Chris Stock ${ }^{\mathrm{a} *}$
}

\begin{abstract}
${ }^{\mathbf{a}}$ School of Physics and Astronomy, University of Edinburgh, Edinburgh EH9 3JZ, United Kingdom, ${ }^{\mathbf{b}}$ School of Chemistry, University of Edinburgh, Edinburgh EH9 3FJ, United Kingdom, and 'ISIS Pulsed Neutron and Muon Source, STFC Rutherford Appleton Laboratory, Harwell Campus, Didcot, Oxon OX11 0QX, United Kingdom. *Correspondence e-mail: cstock@ed.ac.uk
\end{abstract}

An instrument and software algorithm are described for the purpose of characterization of large single crystals at the Alignment Facility of the ISIS spallation neutron source. A method for both characterizing the quality of the sample and aligning it in a particular scattering plane is introduced. A software package written for this instrument is presented, and its utility is demonstrated by way of an example of the structural characterization of large single crystals of $\mathrm{Pb}\left(\mathrm{Mg}_{1 / 3} \mathrm{Nb}_{2 / 3}\right) \mathrm{O}_{3}$. Extensions and modifications of characterization instruments for future improved beamlines are suggested. It is hoped that this software will be used by the neutron community for pre-characterizing large single crystals for spectroscopy experiments and that in the future such a facility will be included as part of the spectroscopy suite at other spallation neutron sources.

\section{Introduction}

Modern developments of neutron chopper spectrometers have resulted in an unprecedented increase in neutron flux at the sample position, along with an increase in angle-resolved detector coverage. Examples of such instrumentation exist at the ISIS neutron and muon spallation source and include MARI, MAPS (Ewings et al., 2019), MERLIN (Bewley et al., 2006) and LET (Bewley et al., 2011). ISIS also hosts a series of backscattering instruments, OSIRIS and IRIS, providing exceptionally high resolution complementing these chopper spectrometers (Telling \& Andersen, 2005; Demmel et al., 2018). All of these instruments have been extremely successful in contributing to solving problems in strongly correlated physics and hence are heavily over-subscribed. Recently developed chopper spectrometers are particularly optimized for use with single-crystal materials, and the prior alignment and characterization of single-crystal samples is crucial to their continued success and for optimal use of facility time.

A key tool for alignment and characterization of single crystals has been Laue X-ray diffraction, which has been applied for both characterization of large single crystals and mapping of grains (Whitley et al., 2015; Chung \& Ice, 1999; Ice $\&$ Pang, 2009). However, a significant problem with laboratory-based Laue diffraction is that X-rays penetrate matter on length scales of the order of micrometres, thus probing only the structure close to the surface. This can lead to misleading or even unhelpful results when characterizing samples with sizes of the order of centimetres. These necessitate the use of neutron scattering, because neutrons interact with materials via nuclear forces and hence are strongly penetrating and probe the bulk structure. 
The use of neutrons to characterize samples before use on modern chopper spectrometers is desirable as alignment and characterization can be time consuming, eating into valuable beamtime. To facilitate this, the excitations group at ISIS maintains a beamline allowing users to align and characterize single crystals beforehand. This is particularly important in experiments using extreme sample environments where alignment of single crystals in a well defined scattering plane is a necessity. Setup and commissioning experiments involving such environments are also time consuming, adding to the 'dead' overhead time of any experiment. The Institut LaueLangevin has likewise recognized the importance of a preexperiment characterization and alignment facility. The OrientExpress beamline (Ouladdiaf et al., 2006), equiped with a neutron Laue camera mounted in a backscattering geometry, is used routinely before diffraction and spectroscopy experiments. We note that this emphasis on the importance of precharacterization has resulted in the development of the larger Cyclops Laue diffractometer (Ouladdiaf et al., 2011).

Here we discuss a methodology of understanding the diffraction data from the alignment facility (ALF) at the ISIS spallation source. ALF has been in operation in some form for approximately 15 years but has recently undergone an upgrade with improved goniometers and more detectors providing wider angular coverage. These upgrades have occurred at the same time as improvements to the chopper instruments, making the facility relevant for users given the increased capabilities at ISIS. As a step before any spectroscopy experiment, it is now important for users to have access to equipment and software that can fully characterize samples in an automated way and visualize the results.

Motivated by this need, we develop a rotation matrix formalism to generate pole figure maps of Bragg peaks in terms of two spherical polar angles and further discuss software developed to visualize data from ALF. We provide demonstration results from a test sample, namely a relaxor ferroelectric, $\mathrm{PbMg}_{1 / 3} \mathrm{Nb}_{2 / 3} \mathrm{O}_{3}$, previously used in spectroscopy measurements (Stock et al., 2018). This approach and software suite were motivated by the application of strain instrumentation and software on the E3 and L3 diffractometers at the now decommissioned NRU reactor (Chalk River, Canada). These instruments and software were often used to co-align samples and fully characterize samples before timeconsuming experiments on the $\mathrm{C} 5$ and N5 triple-axis spectrometers, and indeed the test sample used in this paper has been previously characterized on those diffractometers.

\section{ALF instrument}

ALF was constructed on port N2 of target station 1 at the ISIS spallation neutron source, viewing a liquid methane moderator. The instrument comprises supermirror guides from the source to $8.5 \mathrm{~m}$ of the total flight path, with gaps for ' $t 0$ ' and disc choppers. These choppers produce a pseudowhite beam of neutrons with wavelengths spanning the range from 0.2 to $5.2 \AA$. Thereafter, the instrument flight path is composed of a $\mathrm{B}_{4} \mathrm{C}$ collimation tube through to a sample

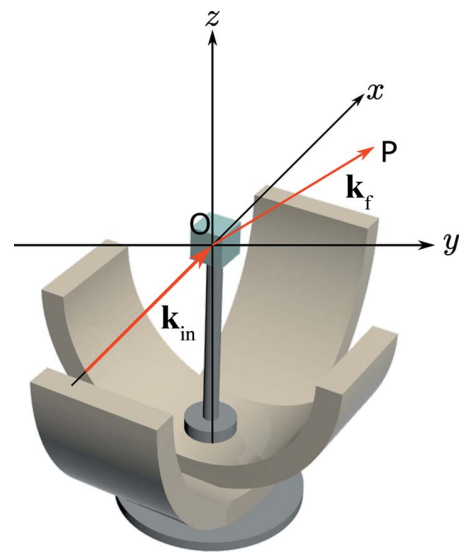

Figure 1

The layout of the goniometer and the definition of the laboratory frame. The red arrows show the scattering of an incoming neutron of wavevector $\mathbf{k}_{\text {in }}$ to a final neutron of wavevector $\mathbf{k}_{\mathrm{f}}$ at pixel $P$.

position situated $14.86 \mathrm{~m}$ from the source. A bank of 37 position-sensitive detector tubes of length $1 \mathrm{~m}$ is situated with its centre point $1.32 \mathrm{~m}$ from the sample, covering a horizontal scattering angle range of $20.2<2 \theta<60.3^{\circ}$. The layout of the current sample position at ALF is illustrated in Fig. 1. The sample position hosts a three-axis goniometer, with adjustable height and position parallel and perpendicular to the incident neutron beam. The lower goniometer rail is aligned along the $x$ axis and the upper is aligned at $90^{\circ}$ along the $y$ axis. These tilt rails are mounted on a rotation stage which rotates these axes around the $z$ axis. In front of the sample, a pseudo-white beam of neutrons is incident on the sample, with the resulting diffraction pattern on the detector being similar to an angularly resolved Laue pattern. Data collection on ALF results in a time-of-flight image of the detector for a given goniometer setting.

This instrument has no monochromatic chopper on the incident side and no neutron wavelength analysers (such as pyrolytic graphite crystals) on the scattered side. In this work, we therefore assume that all measured neutrons on the detector are elastically scattered, transfering no energy to the sample with the momentum transfer defined as $\mathbf{Q}=\mathbf{k}_{\mathrm{f}}-\mathbf{k}_{\mathrm{in}}$. The elastic scattering condition implies that $E_{\text {in }} \equiv E_{\mathrm{f}}$, leading to $\left|\mathbf{k}_{\text {in }}\right| \equiv\left|\mathbf{k}_{\mathrm{f}}\right|$. The energy of a given detected neutron then gives the magnitude of the scattered and incident wavevectors via $E=\hbar^{2} k^{2} / 2 m=\frac{1}{2} m v^{2}=\frac{1}{2} m(l / t)^{2}$, where $\hbar$ is Planck's constant, $m$ the neutron mass, $t$ the time of flight at the detector and $l$ the distance travelled from the target. The magnitude of the momentum transfer is then given via Bragg's law: $|\mathbf{Q}|=2\left|\mathbf{k}_{\text {in }}\right| \sin (\sigma)$. The angle $\sigma$ is defined as half of the angle between $\mathbf{k}_{\text {in }}$ and $\mathbf{k}_{\mathrm{f}}$. Under the assumption of elastic-only scattering, the recorded time of flight of a neutron detected on a pixel of the position-sensitive detector therefore defines the momentum transfer.

\section{Data visualization and the diffraction equations}

Having stated the kinematics and the assumptions of ALF, we now discuss a methodology to visualize the data for a series of 
detector images taken at different goniometer settings. First, as shown in Fig. 2, we consider an Ewald sphere of radius $\left|\mathbf{k}_{\text {in }}\right|$, centred on the crystal. The definition of $x$ and $y$ axes is the same as in Fig. 1. We denote the intersections of $\mathbf{k}_{\text {in }}$ and $\mathbf{k}_{\mathrm{f}}$ with the sphere as the points $N$ and $M$, respectively. According to Bragg's law, the normal of the plane from which neutrons get diffracted bisects $-\mathbf{k}_{\text {in }}$ and $\mathbf{k}_{f}$. We can identify the midpoint of $\mathbf{N M}$ as the point $G$. Since $\mathbf{O G}$ bisects $\mathbf{O N}$ and $\mathbf{O M}$, we can use the location of $G$ to indicate the orientation of the atomic plane.

Because $\mathbf{O G}$ is always perpendicular to the atomic plane from which the diffraction occurs, its location relative to the crystal is fixed. In other words, it rotates with the crystal. A rotation of the crystal about the three-axis relative to the laboratory frame (Fig. 1) can be described by three rotation matrices, representing the crystal's rotation around its base and along the lower and upper goniometer rails, respectively:

$$
\underline{G}_{\text {rotated }}=\underline{\underline{R}}_{\text {rot }} \underline{\underline{R}}_{\text {lower }} \underline{\underline{R}}_{\text {upper }} \underline{G}_{\text {lab }}
$$

where $\underline{\underline{R}}_{\mathrm{rot}}, \underline{\underline{R}}_{\text {lower }}$ and $\underline{\underline{R}}_{\text {upper }}$ are defined as

$$
\begin{gathered}
\underline{\underline{R}}_{\mathrm{rot}}=\left[\begin{array}{ccc}
\cos (\lambda) & -\sin (\lambda) & 0 \\
\sin (\lambda) & \cos (\lambda) & 0 \\
0 & 0 & 1
\end{array}\right], \\
\underline{\underline{R}}_{\text {upper }}=\left[\begin{array}{ccc}
1 & 0 & 0 \\
0 & \cos (\mu) & -\sin (\mu) \\
0 & \sin (\mu) & \cos (\mu)
\end{array}\right], \\
\underline{\underline{R}}_{\text {ower }}=\left[\begin{array}{ccc}
\cos (\tau) & 0 & -\sin (\tau) \\
0 & 1 & 0 \\
\sin (\tau) & 0 & \cos (\tau)
\end{array}\right]
\end{gathered}
$$

and $\lambda, \mu$ and $\tau$ indicate the rotational angle of the crystal around the $z, x$ and $y$ axis, respectively. $\underline{G}_{\text {lab }}$ and $\underline{G}_{\text {rotated }}$ are column vectors describing the vector $\mathbf{O G}$, in Cartesian coordinates, of the laboratory and rotated frames, respectively. Note that $\underline{\underline{R}}_{\mathrm{rot}}, \underline{\underline{R}}_{\mathrm{upper}}$ and $\underline{\underline{R}}_{\text {lower }}$ do not commute with each other, and hence their order matters. The order in which we applied those matrices in equation (1) can be justified by Fig. 3 . It can be seen that any configuration of the crystal can be achieved by first moving the crystal along the upper rail

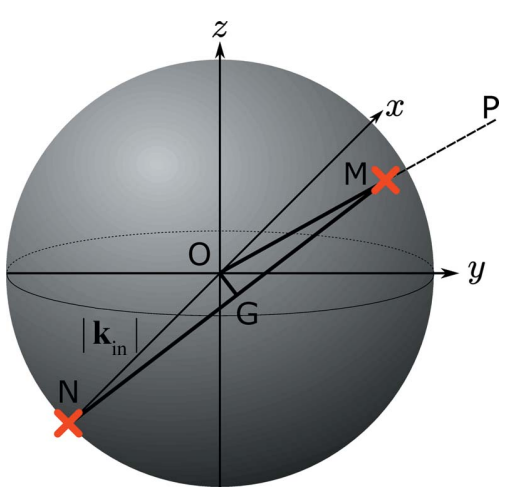

Figure 2

The Ewald sphere whose radius is $\left|\mathbf{k}_{\text {in }}\right|$ centred on the crystal position. OP intersects the sphere at $M$ and the $x$ axis intersects the sphere at $N . G$ is a point on the sphere such that OG bisects $\mathbf{O N}$ and $\mathbf{O M}$. (rotation around the $x$ axis) and then moving the whole upper rail along the lower rail (rotation around the $y$ axis), and finally the base can be rotated to rotate the crystal, along with both goniometer rails, around the $z$ axis.

From each pixel of the detector, we can infer the location of $\underline{G}_{\text {rotated }}$ by geometry (Fig. 2). We can then rotate this vector onto the laboratory frame by systematically inverting the rotation matrices defined in equation (1). For each angular setting illustrated in Fig. 3, data are collected and saved to a NeXus file with the angles and intensity saved using hdf formatting. Application of the above procedure to all files allows those with different gonimeter settings to be mapped onto the same coordinate system.

To begin with, we first need to show how the Cartesian coordinates of $\underline{G}_{\text {rotated }}$ can be deduced from each pixel's location. The pixels' coordinates, $P$, are defined by the three parameters $(l, \alpha, \beta)$, where $l$ is the distance between the pixel and crystal, and $\alpha$ and $\beta$ are the rotational angles around the $x$ and $z$ axes, respectively. The pixel location is thus defined as

$$
\underline{P}=\underline{\underline{A}} \underline{\underline{B}}\left(\begin{array}{l}
l \\
0 \\
0
\end{array}\right),
$$

where

$$
\underline{\underline{A}}=\left[\begin{array}{ccc}
1 & 0 & 0 \\
0 & \cos (\alpha) & -\sin (\alpha) \\
0 & \sin (\alpha) & \cos (\alpha)
\end{array}\right]
$$
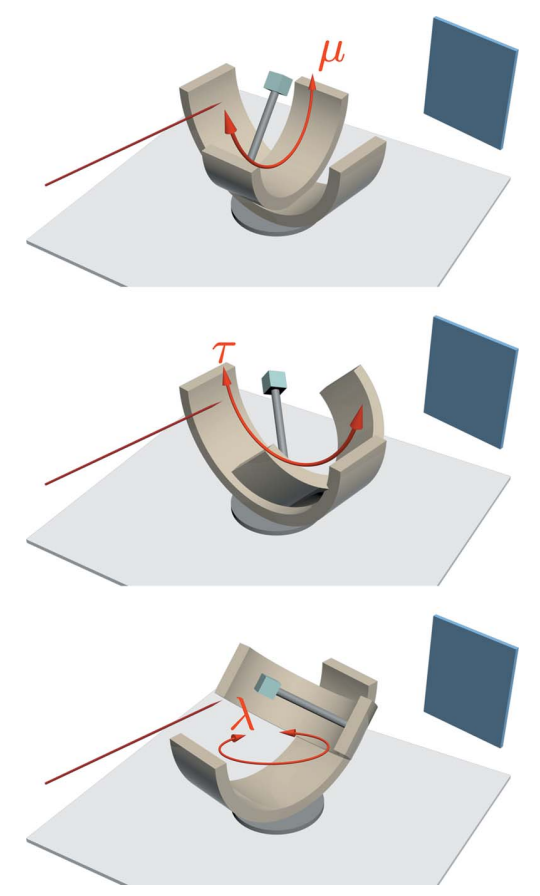

Figure 3

Three frames indicating sequential movement of the goniometer about all three rotational axes. The thin blue cuboid represents the detector bank and the red arrow indicates the path of the incident neutron beam. Accessible sample rotation angles are limited by the angular range of the two goniometer rails. 


$$
\underline{\underline{B}}=\left[\begin{array}{ccc}
\cos (\beta) & -\sin (\beta) & 0 \\
\sin (\beta) & \cos (\beta) & 0 \\
0 & 0 & 1
\end{array}\right] .
$$

The Cartesian coordinates of $\underline{G}_{\text {rotated }}$ can be calculated from the geometric relation between $\underline{G}_{\text {rotated }}$ and $P$. Because $\mathbf{O G}$ and $\mathbf{O P}$ lie in the same plane, $G$ can be mapped onto the $x y$ plane by a rotation by angle $\alpha$, clockwise about the $x$ axis. Since $\mathbf{O G}$ bisects $\mathbf{O N}$ and $\mathbf{O M}$, a further anticlockwise rotation of angle $\beta / 2$ about the $z$ axis aligns OG with the $y$ axis.

That is to say

$$
\underline{\underline{G}}_{\mathrm{rotated}}=\underline{\underline{A}} \underline{\underline{\Omega}}\left(\begin{array}{l}
0 \\
1 \\
0
\end{array}\right),
$$

where

$$
\underline{\underline{\Omega}}=\left[\begin{array}{ccc}
\cos (\beta / 2) & -\sin (\beta / 2) & 0 \\
\sin (\beta / 2) & \cos (\beta / 2) & 0 \\
0 & 0 & 1
\end{array}\right] .
$$

Finally, combining equations (9) and (1), the locations of $\underline{G}_{\text {lab }}$ can be written as

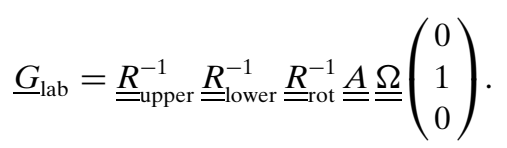

In defining the rotation matrices on the right-hand side of equation (10), five angles are needed, corresponding to the three goniometer angles and the two angles that define the pixel location. However, $\underline{G}_{\text {lab }}$ is uniquely defined by two spherical coordinates, $\theta$ and $\phi$, of the laboratory frame. Therefore, by scanning the five angles provided, the resulting data can be visualized as a pole figure in the form of a twodimensional colourmap. We now illustrate the utility of this method by applying it to an exemplar data set collected on ALF with a sample of single-crystal $\mathrm{PMg}_{1 / 3} \mathrm{Nb}_{2 / 3} \mathrm{O}_{3}$.

\section{Graphical user interface description and test results}

We have applied the above formalism, based on rotation matrices, to design and construct a user interface for the ALF diffractometer. The aim of the program is not to control the instrument, as has been implemented for triple-axis spectrometers (Lumsden et al., 2006) and elsewhere for time-of-flight instruments (ISIS, 2015), but to visualize the data sets taken with different goniometer settings. The goal of this program is to provide a way for a user to quickly visualize data systematically taken for a number of goniometer angle settings for the purposes of alignment and characterization. To illustrate this software and the methodology, we have performed a test series of scans of a single crystal of $\mathrm{PbMg}_{1 / 3} \mathrm{Nb}_{2 / 3} \mathrm{O}_{3}$ used in an experiment on MERLIN to map out the soft phonon modes with temperature (Stock et al., 2018).

The graphical user interface (GUI), written in MATLAB, has four tabs to perform different tasks needed for characterization and alignment. The level of analysis achieved in

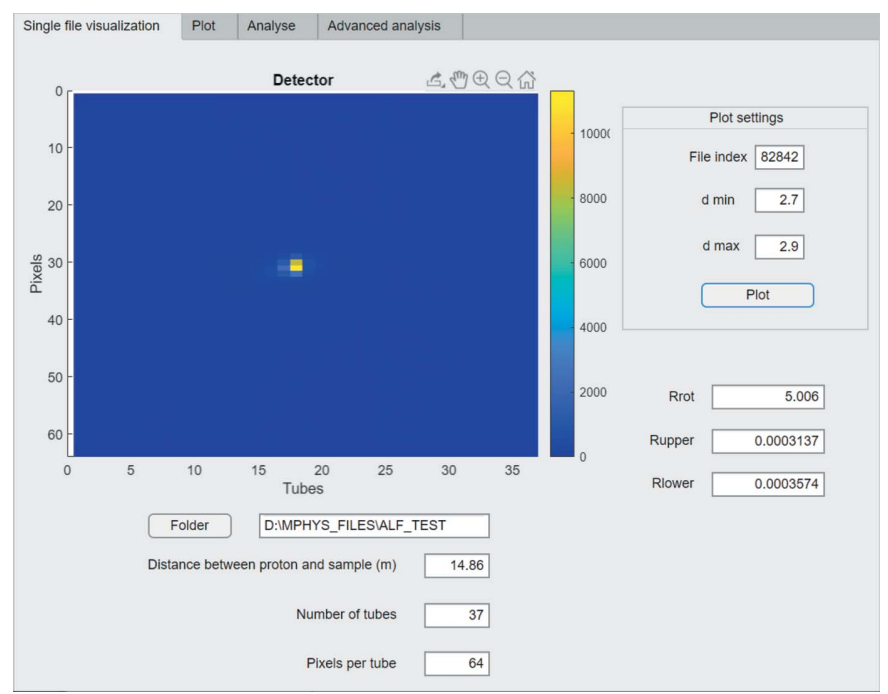

Figure 4

The first tab of the GUI, which is used to visualize the detector from a single input file. The experiment parameters (distance between source and sample, number of tubes, pixels per tube) are auto-completed with the default values for ALF. However, these can be changed if future configurations require it (e.g. increased number of pixels per tube).

each tab gradually increases and is based on the analysis in the previous tabs.

Single-file visualization. The first tab (Fig. 4) is used to visualize the detector bank as the experiment proceeds for a single angular setting of the goniometer illustrated in Fig. 1. The $x$ axis of the figure inside the first tab represents the detector tube index and the $y$ axis represents the pixel index within a given tube. When using this function, the user needs to input the number of the file to be analysed and the $d$-spacing range for the Bragg peak defining the Miller planes of interest for visualization. The GUI will then plot the normalized count rate on the detector bank as a function of $x$ and $y$, and write the rotational angles $\operatorname{Rrot}(\lambda)$, Rupper $(\mu)$ and Rlower $(\tau)$ defining the goniometer angles of the input file.

Plot. The second tab (Fig. 5) enables the user to control how to map all the $\underline{G}_{\text {rotated }}$ files to the laboratory frame. In the experiment setup part, a user can determine whether to add an offset angle to Rrot. This step is necessary as in some experiments the two rails in Fig. 1 may not be initially aligned with the $x$ and $y$ axes used to define the rotation matrices in the section above. Also, since the pixels at the extreme edges of the detector tubes are subject to large read-out errors due to reduced efficiency, the user has the option to mask a certain number of pixels at each end of the tube to increase the quality of data and reduce contributing noise and error. The dropdown resolution menu lets the user choose the angular distance between two adjacent points in $\theta$ and $\phi$ to plot. This defines the angular range over which the data are binned and can be tuned to increase the plotted angular resolution or to increase statistics by coarsening the angular resolution in the output plot. The user can therefore either choose a lower resolution to speed up the plotting process by coarsening the 


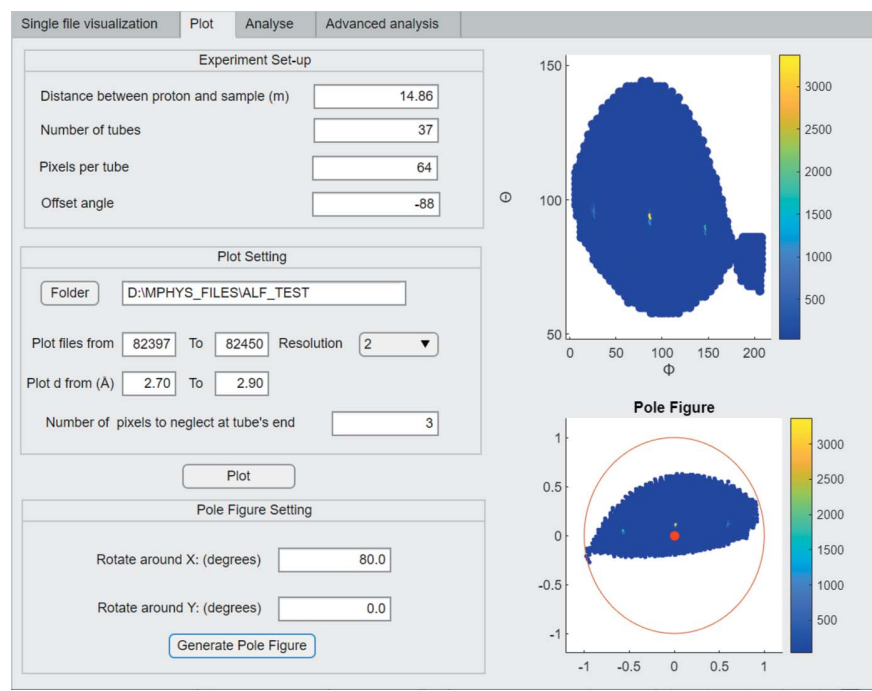

Figure 5

The second tab of the GUI, to map all the peaks to the laboratory frame and generate a pole figure.

plotted angular steps or a higher setting for improved precision and visualization of grains or crystal mosaic.

After all of the $\underline{G}_{\text {rotated }}$ pixels have been mapped to the laboratory frame, the corresponding pole figure can also be generated. The pole figure pieces together all of the different detector images collected at different goniometer settings and plots them onto a common coordinate frame. An Ewald sphere of fixed radius can be then be visualized in spherical coordinates based on two angles. The projection of $\underline{G}_{\text {rotated }}$ onto the equatorial plane is designed to be the intersection of the line connecting $\underline{G}_{\text {rotated }}$ and the south pole. Therefore, if some points are in the southern hemisphere $\left(\theta>90^{\circ}\right)$ then all the points need to be mapped around the $x$ or $y$ axis for an angle determined by the user to avoid any loss of information and clarity in plotting.

Analyse. Then, in the third tab (Fig. 6), some basic analysis can be performed. After loading the $\theta$ and $\phi$ plot generated in the second tab, the user can zoom in and click the figure to get the coordinates of a point. Those coordinates can be put into the GUI to calculate the angle between them. This step can be used to examine whether two peaks are of the same grain by comparing the angle with the theoretical prediction, and will be helpful for grain classification later in the fourth tab.

In circumstances where the user thinks there is a spurious point in the pole figure, they can input the range of $\theta$ and $\phi$ of the region of interest and the GUI will return a list of all the files involved, which could be used for further debugging or remeasurement.

Advanced analysis. The fourth tab (Fig. 7) is divided into two parts. The first is designed to estimate the mosaic angle of the crystal, defined to be the width of a Gaussian fit to the peak shape. After loading the plot generated in the second tab, the user can move the sliders to create two lines of constant $\theta$ and $\phi$, respectively. Then a constant $\theta$ or $\phi$ cut can be plotted. The origin is the intersection of the two lines, and this figure changes in real time when the user moves the

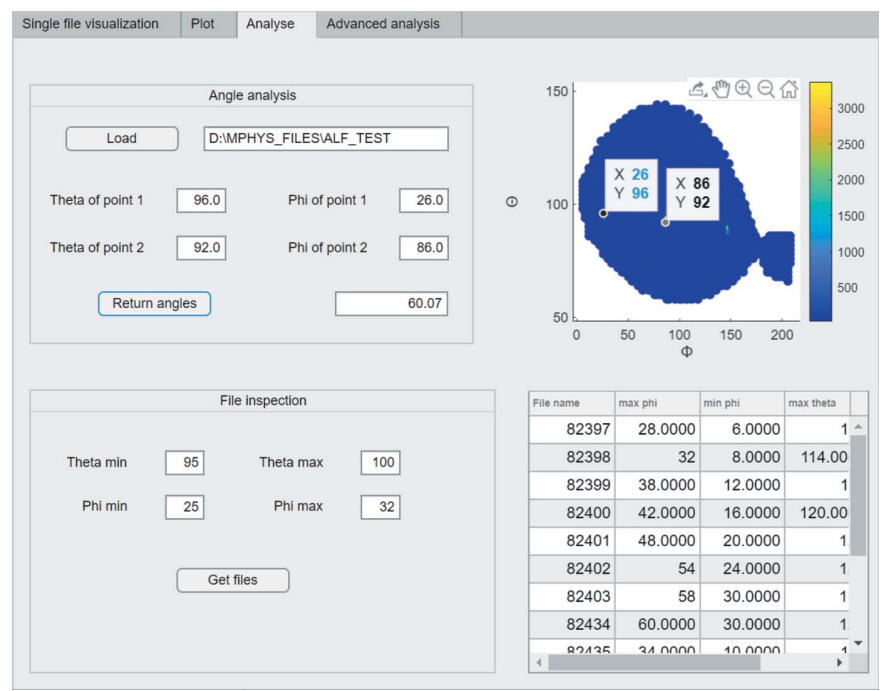

Figure 6

The third tab of the GUI. Angles between two peaks can be calculated and files containing points within the region of interest can be traced.
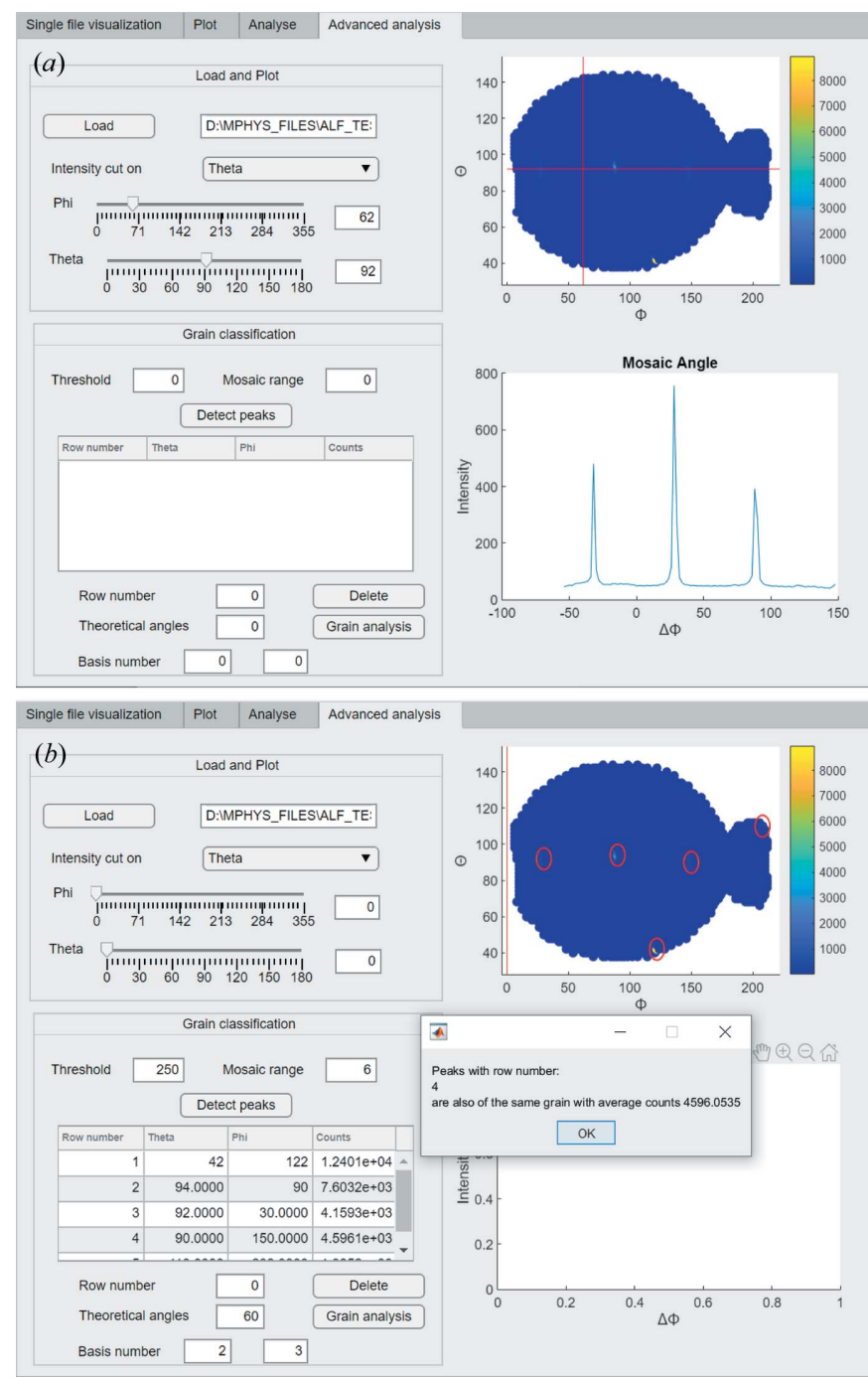

Figure 7

The fourth tab of the GUI. Part ( $a$ ) is used to determine the mosaic angle of each peak along $\phi$ or $\theta$. Part $(b)$ is a tool that allows for grain classification. 
sliders, providing a rapid way to characterize the crystal quality and understand the pole figure.

The second part is used to determine how many grains a sample has and an estimate of the percentage of the total that each makes up. To do this, we first let the GUI detect and mark all the peaks with neutron counts higher than the user-defined threshold. Then, the user inputs a mosaic range so that any points deviating from the peak's location within this range are considered to be part of the peak when summing the neutron counts.

The GUI will then print a list of all the peaks detected with their locations in $(\theta, \phi)$ and the corresponding integrated neutron counts. From this information, the user must select two peaks as bases that they know to be of the same grain. This step is necessary because at least two peaks are needed to uniquely define the location of a grain. Then, the user can input the theoretical angular separation of two adjacent peaks from the same grain and let the GUI search the list for peaks of the same grain.

Once the GUI returns its search results the user will know which peaks in the plot are of the same grain and the average neutron counts of those peaks. The user can then take a note of the locations and the grain's average neutron counts before deleting them from the list. In cases where the neutron counts in a peak come from multiple grains, the user must neglect this peak and use the average neutron counts from other peaks of the same grain instead. This process can be done several times until all the peaks in the list have been identified. At the end of this process, the locations of each grain will be known and the ratio of the neutron counts from one type of grain to the total neutron counts detected can be taken as the approximate percentage of the prevalence of this grain in the crystal.

\section{Conclusions}

The present paper has shown the utility of using ALF for characterization and subsequent alignment of large single crystals. One thing that is evident from the pole figures constructed in this test experiment is that much of the angular range is not accessible in the current configuration of ALF. A means of improving the facility for future and more complete characterization of single crystals is the use of an Eularian cradle. In this case, the extension of the software discussed here would be to replace the two tilt matrices listed above $\left(\underline{\underline{R}}_{\text {lower }}, \underline{\underline{R}}_{\text {upper }}\right)$ by a single rotation matrix and include an inner rotation matrix. The series of rotation matrices is described by Busing \& Levy (1967). However, in the current instrumental configuration on ALF, through a combined scan of the tilt axes and rotation (Fig. 1) axes, much of the Ewald sphere can be measured to characterize the quality of the crystal and to perform alignment for measurement on more advanced instruments. We hope that by providing this methodology and outline we will allow future excitations users to take advantage of this code and GUI, and that other spallation source facilities will support similar instruments to improve use of more complex chopper instruments.

\section{Acknowledgements}

We thank J. A. Rodriguez and P. M. Gehring at NIST for helpful discussions. The authors are also grateful to the neutron scattering group at Chalk River, in particular Z. Tun, W. J. L. Buyers and I. P. Swainson, for their help and support in many experiments at the NRU reactor that provided the motivation for this project.

\section{Funding information}

We thank the EPSRC and the STFC for funding. HL was cofunded by the ISIS facility development studentship programme.

\section{References}

Bewley, R. I., Eccleston, R. S., McEwen, K. A., Hayden, S. M., Dove, M. T., Bennington, S. M., Treadgold, J. R. \& Coleman, R. L. S. (2006). Physica B, 385-386, 1029-1031.

Bewley, R. I., Taylor, J. W. \& Bennington, S. M. (2011). Nucl. Instrum. Methods Phys. Res. A, 637, 128-134.

Busing, W. R. \& Levy, H. A. (1967). Acta Cryst. 22, 457-464.

Chung, J. S. \& Ice, G. E. (1999). J. Appl. Phys. 86, 5249-5255.

Demmel, F., McPhail, D., French, C., Maxwell, D., Harrison, S., Boxall, J., Rhodes, N., Mukhopadhyay, S., Silverwood, I., Sakai, V. G. \& Fernandez-Alonso, F. (2018). J. Phys. Conf. Ser. 1021, 012027.

Ewings, R. A., Stewart, J. R., Perring, T. G., Bewley, R. I., Le, M. D., Raspino, D., Pooley, D. E., Śkoro, G., Waller, S. P., Zacek, D., Smith, C. A. \& Riehl-Shaw, R. C. (2019). Rev. Sci. Instrum. 90, 035110.

Ice, J. E. \& Pang, J. W. L. (2009). Mater. Charact. 60, 1191-1201.

ISIS (2015). IBEX, https://www.isis.stfc.ac.uk/Pages/IBEX.aspx.

Lumsden, M. D., Robertson, J. L. \& Yethiraj, M. (2006). Physica B, 385-386, 1336-1339.

Ouladdiaf, B., Archer, J., Allibon, J. R., Decarpentrie, P., LeméeCailleau, M.-H., Rodríguez-Carvajal, J., Hewat, A. W., York, S., Brau, D. \& McIntyre, G. J. (2011). J. Appl. Cryst. 44, 392-397.

Ouladdiaf, B., Archer, J., McIntyre, G. J., Hewat, A. W., Brau, D. \& York, S. (2006). Physica B, 385-386, 1052-1054.

Stock, C., Gehring, P. M., Ewings, R. A., Xu, G., Li, J., Viehland, D. \& Luo, H. (2018). Phys. Rev. Mater. 2, 024404.

Telling, M. T. F. \& Andersen, K. H. (2005). Phys. Chem. Chem. Phys. 7, 1255-1261.

Whitley, W., Stock, C. \& Huxley, A. D. (2015). J. Appl. Cryst. 48, $1342-1345$ 